\title{
Effect of Vibration Parameters on Properties of Polypropylene in Dynamic Plasticizing Process
}

\author{
Yan Liu ${ }^{1, a}$, Chuanbo Li ${ }^{1, b}$ \\ ${ }^{1}$ ShanDong Labor Vocational and Technical College, Jinan 250022, China; \\ ${ }^{2}$ ShanDong Labor Vocational and Technical College, Jinan 250022, China. \\ a850056766@qq.com, b362506988@qq.com
}

Keywords: Polypropylene, Plasticizing, Vibration parameters, Energy consumption.

\begin{abstract}
The Polypropylene samples were molded by novel hydraulic vibration injection molding machine. The effects of vibration amplitude and frequency on physical and mechanical properties of PP and power consumption in the plasticizing process were studied. The results show that in the case of imposing vibration under otherwise identical conditions, the tensile strength of PP sample increases by $9.8 \%$, the impact strength increases by $13.8 \%$, the density increases by $0.3 \%$, while the power consumption of the injection molding machine decreases by $6.6 \%$.
\end{abstract}

\section{Introduction}

One of the most common manufacturing techniques for polymer products is injection molding. The screw injection molding machine is main processing equipment. The screw systems are the most key unit because it directly influences plasticizing effect of material and product quality. However, the screw effective length is shorten for the traditional injection molding machine, it makes plasticizing and mixture effect of material is not uniform compared with screw extruder, which affects the product's quality.

With this problem in mind, the concept of vibration-assisted injection molding has been developed[1-2]. The main merit is enable theological behavior of polymer melt. The vibration technique has gained a lot of investigations for polymer processing. Jinping Qu and coworkers [3-8]mainly researched the influence of the electromagnetism vibration on the fill-system. Kaizhi Shen and coworkers[9] had attained fine mechanical properties via using the dynamic packing technique. Above the vibration techniques are introduced into polymer melt only in packing pressure, Although there has been many researchers about properties of polymer via using the vibration techniques, such as effect of vibration parameters on the screw plasticizing is absent. A novel self-designed hydraulic dynamic injection molding machine invented by Jin-ping Qu is another example utilizing vibration technique. In this kind of injection machine, the vibration field is applied to the solid conveying, plasticizing, melting, injection and packing pressure of the entire molding process by the axial vibration of the screw, which vibration parameters of each process can be adjusted separately. The novel injection molding machine has significant advantages, such as low energy consumption, low injection pressure and melt apparent viscosity compared with the conventional injection molding machine.

The purpose of the work is to study the influencing regulation of properties of Polypropylene by changing vibration parameters only imposed on plasticizing process.

\section{Experimental}

\subsection{Materials}

The sample material used in this work was polypropylene (PP) in the form of pellets and with a trademark 1100NK (MFI=11g/10min), spinning grade, supplied by the Thai Petrochemical Ind Co.,Ltd, Thailand. 


\subsection{Apparatus}

The apparatus used in this experiment was a self-made hydraulic dynamic injection molding machine (model HD-98). The novel injection molding machine is different from the traditional injection machine. The axially pulsed screw can be achieved by back pressure valve superposing a rectangular current signal, thus the whole plasticizing process can be accomplished in pulsed pressure. The vibration amplitude and frequency can be adjusted independently by controlling signal of back pressure valve. The diameter of screw is $32 \mathrm{~mm}$, the $\mathrm{L} / \mathrm{D}$ ratio is $24.8: 1$ and the clamping force is $980 \mathrm{KN}$.

\subsection{Sample preparation}

The samples of mechanical tests were using the formula cooled to the room temperature $\left(23^{\circ} \mathrm{C}\right)$ in air. The mold temperature was controlled at $40^{\circ} \mathrm{C}$ through a thermolator that supplied heated water to the mold in a closed loop network of hoses. The experiments were performed at a range of vibration parameters.

\subsection{Testing}

The universal tensile testing machine (Model INSTRON 5566, INSTRON corp. American) was used for tensile testing at room temperature $\left(23^{\circ} \mathrm{C}\right)$, at a crosshead speed of $50 \mathrm{~mm} / \mathrm{min}$. The pendulum impact testing machine (Model POE2000, INSTRON corp. American) was used for impact testing. The thermal analyses were performed using a differential scanning calorimeter (NETZSCH DSC-204) The temperature test range is from $-170^{\circ} \mathrm{C}$ to $730^{\circ} \mathrm{C}$ and temperature accuracy is $0.01^{\circ} \mathrm{C}$. The samples were heated at a rate of $10^{\circ} \mathrm{C}$ in the temperature range of $25-220^{\circ} \mathrm{C}$ in a nitrogen atmosphere. Each sample was weighed approximately $5 \mathrm{mg}$. All results were recorded for the first heating of the samples. The digital energy consumption instrument (Model WT1600, Yokogawa corp. Japan) was used for testing energy consumption.

\section{Results and discussion}

\subsection{Mechanical properties}

"A" and "f" represents the vibration amplitude and vibration frequency respectively in the paper. Figure. 1 shows the effect of vibration amplitude on tensile strength in different frequency.

The experimental results show the tensile stress is improved with increase of vibration amplitude keeping the same frequency in plasticizing process, and then, the tensile stress decreases slightly with increase of the vibration amplitude further. When vibration amplitude is less, the increasing extent of tensile stress is sharp. The tensile stress reaches to max value quickly at $\mathrm{f}=4 \mathrm{~Hz}$. This expresses the tensile stress is most sensitive at $\mathrm{f}=4 \mathrm{~Hz}$. The maximum increment is $9.8 \%$, from $32.4 \mathrm{MPa}$ of the steady sample to $35.6 \mathrm{MPa}$ of the sample obtained at $\mathrm{f}=4 \mathrm{~Hz}$ and $\mathrm{A}=0.4 \mathrm{~mm}$. The tensile stresses of all dynamic samples are improved than that of no vibration samples.

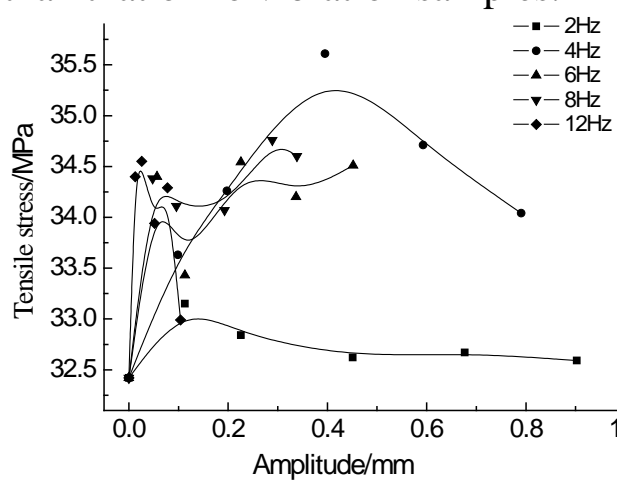

Figure.1 Tensile strength of PP samples under different frequencies and vibration amplitudes

Figure 2 for the PP sample impact strength under different frequency amplitude changes with the curve. When the frequency is constant, with the increase of amplitude, PP sample after impact strength to rise sharply declined slightly. For different frequency rate, there is a best maximize the 
impact strength value of amplitude, low frequency occurs in strong amplitude, high frequency occurs in amplitude is relatively small, and at the age of $8 \mathrm{~Hz}$ frequency, the impact strength of PP sample, the biggest increase by $13.8 \%$.

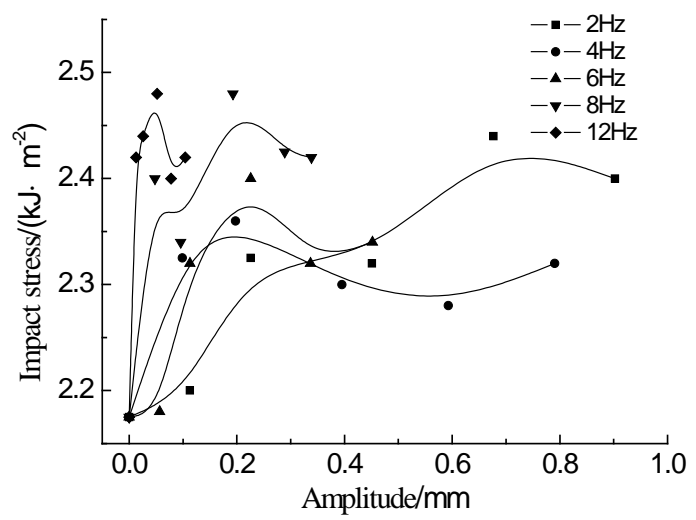

Figure.2 Impact strength of PP samples under different frequencies and vibration amplitudes

\subsection{Density of sample}

Figure 3 for the PP sample density changing with the amplitude under different frequency curve. As can be seen from the figure 4, the density of various vibration conditions PP sample were greater than under steady molding sample density. Is applied to each vibration frequency, vibration significantly increased in density value, and then under the same frequency with the increase of amplitude, slow decline after the peak. With the increase of frequency, PP sample density required for maximum amplitude decreases, which in the $4 \mathrm{~Hz}$ frequency, amplitude of $0.1 \mathrm{~mm}$, PP sample density increase, the biggest increase by about $3 \%$.

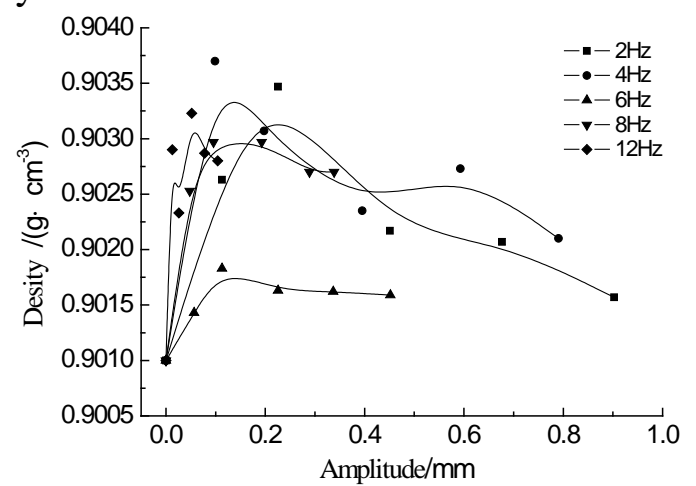

Figure.3 Density of PP samples under different frequencies and vibration amplitudes

\subsection{DSC test}

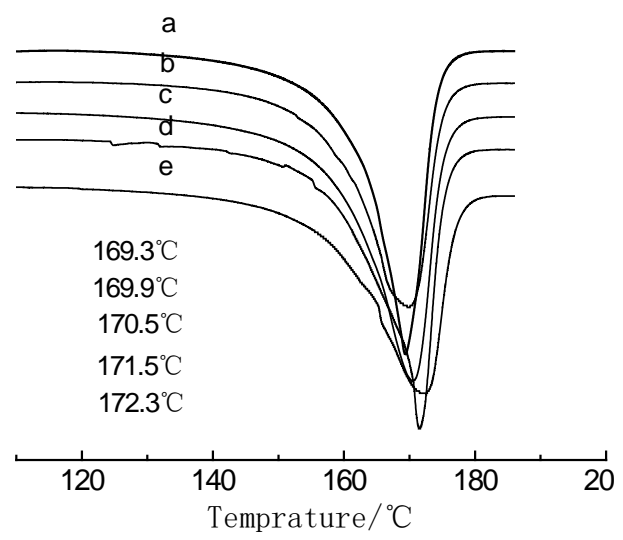

Figure.4 DSC curves of PP samples under different vibration amplitudes

Figure 4 is the frequency of $4 \mathrm{~Hz}$ when PP sample under different amplitude of DSC curve, the data listed in table 1. More steady molding samples with dynamic injection molding samples, we can see the steady-state sample melting peak is relatively narrow, as shown in the curve a, melting point is 
169. $3{ }^{\circ} \mathrm{C}$. Can be seen by the curve b e, dynamic injection molding samples, with the increase of amplitude plasticizing process screw melting peak width, moving in the direction of high temperature melting point at the same time.

Table.1 DSC testing results of PP samples under different vibration amplitudes

\begin{tabular}{cccc}
\hline amplitude $/ \mathrm{mm}$ melt point $/{ }^{\circ} \mathrm{C}$ & $\triangle \mathrm{Hf} /(\mathrm{J} \cdot \mathrm{g}-1)$ & crystallization $/ \%$ \\
\hline 0 & 169.3 & 87.71 & 41.97 \\
0.1 & 169.9 & 87.84 & 42.03 \\
0.19 & 170.5 & 92.68 & 44.34 \\
0.4 & 171.5 & 92.32 & 44.17 \\
0.65 & 172.3 & 93.24 & 44.61
\end{tabular}

Can be seen from Table 1, fluctuating pressure induced junction in the molding sample crystal degrees higher than steady molding. This is because in the process of dynamic plasticizing, plasticizing, melting effect is good, to make points better child orientation in injection molding, is conducive to the formation of ice crystals, and crystallization increased. With the increase of amplitude, the melt by the shearing action of enhancement, thus present a trend of increasing crystallization.

\subsection{Energy consumption}

To study the hydraulic pulse forming on the plasticizing process of vibration to the influence of consumption, from the total input power injection machine access digital power analysis instrument, the other parameters unchanged, by changing the amplitude and frequency of the plasticizing process, analyzed the influence of vibration parameters on the energy consumption of the whole machine.

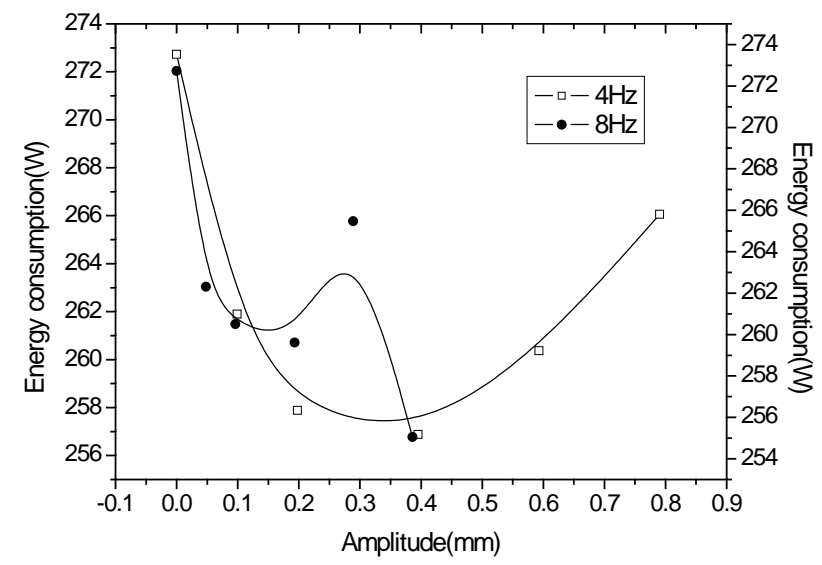

Figure.5 Power consumption of PP under different frequencies and vibration amplitudes

Figure 5 is the total molding curved line along with the change of amplitude of plasticizing process energy consumption. When the frequency of $4 \mathrm{~Hz}$, with the increase of amplitude, rise, after the whole processing to reduce the energy consumption when amplitude increases to $0.4 \mathrm{~mm}$, to achieve the minimum energy consumption. When the frequency is $8 \mathrm{~Hz}$, within the scope of the experiment, when the amplitude increases to $0.4 \mathrm{~mm}$, the whole processing of reducing energy consumption of about $6.6 \%$.

Under the fluctuating pressure generated by the plasticizing process of shear heat dissipation Reduce the apparent viscosity of the polymer melt, plasticizing of screw driver work rate and injection molding of resistance will be reduced, so the same processing conditions, forming the overall energy consumption decreased.

\section{Summary}

Compared to the conventional injection molding, the tensile stress, impact stress and density of PP samples are improved a certain extent using the novel hydraulic dynamic injection molding machine due to pulsed pressure induced in plasticizing process. The melt point of samples rises, which facilitates the perfection of crystal. Furthermore, the whole molding resistance decreased by dynamic 
plasticizing, the general energy consumption dropped. So, for the novel hydraulic dynamic injection molding machine, the plasticizing and mixture can be improved by vibration introduced in plasticizing process.

\section{References}

[1] Ibar J P. Control of polymer properties by melt vibration technology: a review[J]. Polymer Engineering Science, 1998, 38(1):1-20.

[2] Bevis M J, et al. Processing and physical property relationship in injection-molded isotatic polypropylene.[J]. Journal of Polymer Science, Part B: Polymer Physics, 1997,35(2): 241-263.

[3] Wang Quan, Qu Jinping. Effect of Vibration Parameters on Plasticizing Capability of Dynamic Injection Screw [J]. Plastics Science and Technology, 2009,37(7): 69-72.

[4] Wang Xishun, Peng Yucheng, Chen Qili, et al. Application of Oscillation Technology in Injection Molding [J]. CHINA PLASTICS, 1999, 13(4): 49-54.

[5] Wang Quan, Qu Jinping. Study on Rheological Behaviors of Calcium Carbonate-Filled Polypropylene in Dynamic Processing [J]. Polymer Materials Science \& Engineering, 2007, 23(1): 176-179.

[6] Quan Wang , Jinping Qu. Effect of vibration parameters of electromagnetic dynamic plastics injection molding machine on mechanical properties of polypropylene samples[J]. Journal of Applied Polymer Science, 2006,102(2): 972-976.

[7] Peng Xiangfang, Liu Ting, Xu Chao, et al. Research on the Effect of Whole-Course Vibration Injection Molding on Mechanical Properties of PP [J]. Engineering Plastics Application, 2004, 32(11): 25-27.

[8] Qu Jin ping,Wu Hong wu. New Technology of Dynamic Vibration Field Enhancing Polymer Injection Molding [J]. Plastics, 2000, 29(6): 9-12.

[9] Guan Qing, Shen Kaizhi, et al. Self-reinforcement of polypropylene by oscillating packing injection molding under low pressure[J]. Journal of Applied Polymer Science, 1996, 62: 755-762. 